Kragujevac Journal of Mathematics

Volume 40(1) (2016), Pages 73-78.

\title{
ON A CONJECTURE OF HARMONIC INDEX AND DIAMETER OF GRAPHS
}

\section{J. AMALORPAVA JERLINE ${ }^{1}$ AND L. BENEDICT MICHAELRAJ ${ }^{2}$}

Abstract. The Harmonic index $H(G)$ of a graph $G$ is defined as the sum of the weights $\frac{2}{d(u)+d(v)}$ of all edges $u v$ of $G$, where $d(u)$ denotes the degree of the vertex $u$ in $G$. In this work, we prove the conjecture $H(G)-D(G) \geq \frac{5}{6}-\frac{n}{2}$ given by Liu in 2013 when $\mathrm{G}$ is a unicyclic graph by giving a better bound, namely, $H(G)-D(G) \geq \frac{5}{3}-\frac{n}{2}$.

\section{INTRODUCTION}

Let $G=(V, E)$ be a simple connected graph with vertex set $V(G)$ and edge set $E(G)$. For a graph $G$, the harmonic index $H(G)$ is defined as $H(G)=\sum_{u v \in E(G)} \frac{2}{d(u)+d(v)}$, where $d(u)$ is the degree of the vertex $u$ in $G$. As far as we know, this index first appeared in [3]. Zhong found the minimum and maximum values of the harmonic index for simple connected graphs, trees and unicyclic graphs and characterized the corresponding extremal graphs [6,7]. Wu et al. gave a best possible lower bound for the harmonic index of triangle-free graph with minimum degree at least two and characterized the extremal graphs [5]. Deng et al. considered the relation connecting the harmonic index $H(G)$ and the chromatic number $\chi(G)$ and proved that $\chi(G) \leq$ $2 H(G)$ by using the effect of removal of a minimum degree vertex on the harmonic index [2]. Liu proved that $H(T)-D(T) \geq \frac{5}{6}-\frac{n}{2}$ and $\frac{H(T)}{D(T)} \geq \frac{1}{2}+\frac{1}{3(n-1)}$ for trees with equality for path and propose it as a conjecture for any connected graph of order $n$

Key words and phrases. Harmonic index, diameter, unicyclic graph.

2010 Mathematics Subject Classification. Primary: 05C07. Secondary: 05C12.

Received: November 12, 2015.

Accepted: January 29, 2016. 


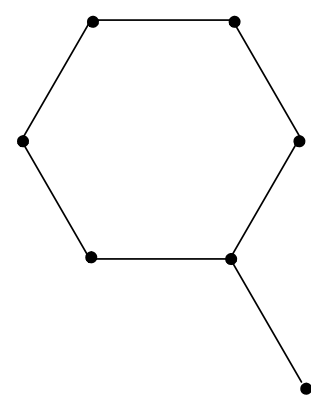

FiguRE 1. $U_{7}^{1}$

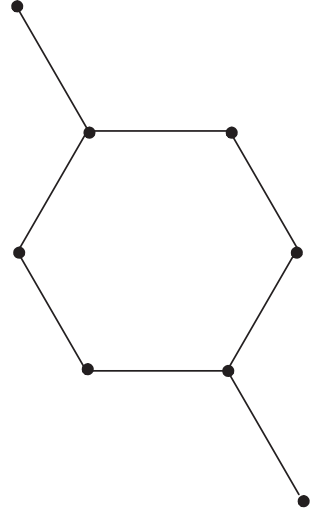

(A) $U_{8}^{2}$

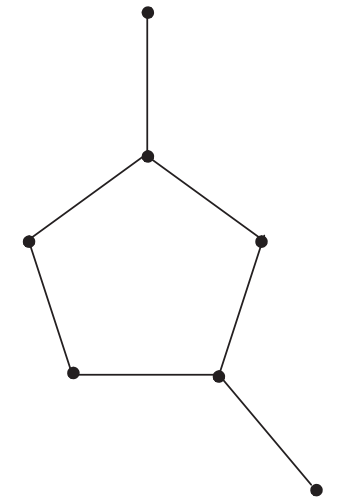

(в) $U_{7}^{2}$

FiguRE 2.

[4]. In this work, we prove the conjecture when $G$ is an unicyclic graph by giving a better bound, namely, $H(G)-D(G) \geq \frac{5}{3}-\frac{n}{2}$ for $n \geq 7$.

We conclude this section with some notations and terminology. Let $G=(V, E)$ be a simple connected graph with vertex set $V(G)$ and edge set $E(G)$. The degree of a vertex $v$ of $G$ is denoted by $d(v)$. If $d(v)=1$, then $v$ is said to be a pendant vertex of $G$. The edge incident with the pendant vertex $v$ is referred to as pendant edge and the vertex adjacent to $v$ is referred as the support vertex of $v$. The set of neighbours of $v$ is denoted by $N(v)$. The eccentricity of a vertex $v$ is the greatest distance from $v$ to any other vertex of $G$. The diameter of a graph is the maximum over eccentricities of all vertices of the graph and it is denoted by $D(G)$. A diametrical path of a graph is a shortest path whose length is equal to the diameter of the graph. As usual, $C_{n}$ denotes the cycle on $n$ vertices. Let $U_{n}^{1}$ be a graph obtained from $C_{n-1}$ by attaching one pendant edge to any one of the vertices of $C_{n-1}$ and $U_{n}^{2}$ be a graph obtained from $C_{n-2}$ by attaching two pendant edges to two vertices of $C_{n-2}$, say $u$ and $v$, such that $d(u, v)=\frac{n-2}{2}$, if $n$ is even and $d(u, v)=\frac{n-3}{2}$, if $n$ is odd (see Figures 1 and 2). A unicyclic graph is odd or even, if the number of vertices in its cycle is odd or even. For other notations in graph theory, may be consulted [1]. 


\section{BASIC RESUltS}

In this section we have some observations and lemmas which are used to prove our main result. Analysing diametrical paths of the unicyclic graphs, we have the following observations.

1. Let $G ¥ C_{n}$ be a unicyclic graph on $n$ vertices. Then at least one of the end vertices of a diametrical path of $G$ must be a pendant vertex.

2. Let $G ¥ C_{n}$ be a unicyclic graph on $n$ vertices and $v$ be an end vertex in a diametrical path of $G$. If $D(G-v)=D(G)-1$, then $v$ must lie on every diametrical path of $G$.

3. Let $G ¥ C_{n}$ be a unicyclic graph on $n$ vertices. If $v$ is a pendant vertex of a diametrical path and $u$ is the support vertex of $v$, then $N(u)$ has at most two vertices of degree at least 2 .

Lemma 2.1. $f(x)=\frac{2}{x+2}-\frac{2}{x+1}$ is an increasing function for $x>0$.

Lemma 2.2. Let $v$ be a pendant vertex of a connected graph $G$. Then $H(G) \geq$ $H(G-v)$.

Proof. Let $u$ be the support vertex of $v$. Then

$$
\begin{aligned}
H(G)-H(G-v) & =\frac{2}{d(u)+1}+2 \sum_{w \in N(u)-\{v\}}\left(\frac{1}{d(u)+d(w)}-\frac{1}{d(u)+d(w)-1}\right) \\
& \geq \frac{2}{d(u)+1}+2(d(u)-1)\left(\frac{1}{d(u)+1}-\frac{1}{d(u)}\right)\{\text { by Lemma } 2.1\} \\
& \geq 0 .
\end{aligned}
$$

Hence $H(G) \geq H(G-v)$.

Lemma 2.3. Let $G$ be a unicyclic graph of order $n \geq 7$ with $1 \leq n_{1} \leq n-3$ pendant vertices, $v$ be an end vertex of a diametrical path of $G$ such that $v$ is a pendant vertex of $G$ and $u$ be the support vertex of $v$. If $N(u)$ has exactly one vertex of degree $\geq 2$ and $D(G-v)=D(G)-1$, then $H(G)-H(G-v) \geq \frac{1}{2}$.

Proof. In this case $d(u)=2$, otherwise $u$ has at least one more pendant neighbour contradicting $D(G-v)=D(G)-1$. If the other neighbour of $u$ is $w$, then

$$
H(G)-H(G-v)=\frac{2}{2+1}+\left\{\frac{2}{2+d(w)}-\frac{2}{1+d(w)}\right\} .
$$

By Lemma 2.1 and $d(w) \geq 2$,

$$
H(G)-H(G-v) \geq \frac{2}{3}+\left\{\frac{2}{2+2}-\frac{2}{1+2}\right\}=\frac{1}{2} .
$$

Lemma 2.4. Let $G$ be a unicyclic graph of order $n \geq 7$ with $2 \leq n_{1} \leq n-4$ pendant vertices and $u$ and $v$ be the end vertices of a diametrical path of $G$ such that $u$ and $v$ 
are the pendant vertices. Let $u^{\prime}$ and $v^{\prime}$ be their respective support vertices. If $N\left(u^{\prime}\right)$ and $N\left(v^{\prime}\right)$ each has exactly two vertices of degree $\geq 2$ and $D(G-v)=D(G)-1$, then $H(G)-D(G) \geq \frac{5}{3}-\frac{n}{2}$.

Proof. We have the following cases.

Case 1: If there is no other pendant vertices in $G$ other than $u$ and $v$, then $G$ will be of the form $U_{n}^{2}$. Also $H\left(U_{n}^{2}\right)=\frac{n}{2}-\frac{2}{5}$. If $n$ is even, then $D\left(U_{n}^{2}\right)=\frac{n}{2}+1$. Hence $H\left(U_{n}^{2}\right)-D\left(U_{n}^{2}\right) \geq \frac{-7}{5} \geq \frac{5}{3}-\frac{n}{2}$. If $n$ is odd, then $D\left(U_{n}^{2}\right)=\frac{n}{2}+\frac{1}{2}$. Hence $H\left(U_{n}^{2}\right)-D\left(U_{n}^{2}\right) \geq \frac{-9}{10} \geq \frac{5}{3}-\frac{n}{2}$.

Case 2: If there is at least one pendant vertex in $G$ other than $u$ and $v$, then we delete those pendant vertices one by one until the resulting graph is $U_{n-n_{1}+2}^{2}$. Assume that $v_{1}, v_{2}, \ldots, v_{n_{1}-2}$ are the pendant vertices in the order they are deleted. Therefore by Lemma 2.2 we have $H(G) \geq H\left(G-v_{1}\right) \geq \cdots \geq H\left(G-\bigcup_{i=1}^{n_{1}-2} v_{i}\right)=H\left(U_{n-n_{1}+2}^{2}\right)$ and $D(G)=D\left(G-v_{1}\right)=\cdots=D\left(G-\bigcup_{i=1}^{n_{1}-2} v_{i}\right)=D\left(U_{n-n_{1}+2}^{2}\right)$. Also $H\left(U_{n-n_{1}+2}^{2}\right)=$ $\frac{n-n_{1}+2}{2}-\frac{2}{5}$. If $U_{n-n_{1}+2}^{2}$ is even, then $D\left(U_{n-n_{1}+2}^{2}\right)=\frac{n-n_{1}+2}{2}+1$. Hence $H(G)-D(G) \geq H\left(U_{n-n_{1}+2}^{2}\right)-D\left(U_{n-n_{1}+2}^{2}\right)=\frac{-7}{5} \geq \frac{5}{3}-\frac{n}{2}$. If $U_{n-n_{1}+2}^{2}$ is odd, then $D\left(U_{n-n_{1}+2}^{2}\right)=\frac{n-n_{1}+2}{2}+\frac{1}{2}$. Hence $H(G)-D(G) \geq H\left(U_{n-n_{1}+2}^{2}\right)-D\left(U_{n-n_{1}+2}^{2}\right)=$ $\frac{-9}{10} \geq \frac{5}{3}-\frac{n}{2}$.

\section{Main Result}

In this section, we give a sharp lower bound of the relationship involving the harmonic index and diameter of connected unicyclic graphs. Let $\mathscr{C}$ be a set of graphs obtained from $C_{4}$ by attaching one pendant edge and a path of length $n-5$ to two diametrically nonadjacent vertices of $C_{4}$. For each $n \geq 7$, we have exactly one graph in this set.

Theorem 3.1. Let $G$ be a unicyclic graph of order $n \geq 7$ and diameter $D(G)$. Then $H(G)-D(G) \geq \frac{5}{3}-\frac{n}{2}$, where equality holds if $G \in \mathscr{C}$.

Proof. We have the following cases.

Case 1: Let $G \cong C_{n}$. For $C_{n}, \quad H\left(C_{n}\right)=\frac{n}{2}$. If $n$ is odd, then $D\left(C_{n}\right)=\frac{n-1}{2}$. Therefore $H\left(C_{n}\right)-D\left(C_{n}\right)=\frac{n}{2}-\frac{n-1}{2}=\frac{1}{2} \geq \frac{5}{3}-\frac{n}{2}$. If $n$ is even, then $D\left(C_{n}\right)=\frac{n}{2}$. Therefore $H\left(C_{n}\right)-D\left(C_{n}\right)=\frac{n}{2}-\frac{n}{2}=0 \geq \frac{5}{3}-\frac{n}{2}$ 
Case 2: Let $G ¥ C_{n}$. Then $G$ has at least one pendant vertex. By observation 1, at least one of the end vertices of the diametrical path of $G$ is a pendant vertex, say $v$. Also $D(G-v)=D(G)$ or $D(G-v)=D(G)-1$.

Subcase 2.1: Let $D(G-v)=D(G)$. In this case we can prove the result by induction on $n$. We can easily check for unicyclic graph $G$ of order 7 such that $D(G-v)=D(G)$. Assume the result is true for all unicyclic graphs of order less than or equal to $n-1$. Let $G$ be a unicyclic graph of order $n$ and $v$ be the pendant vertex of the diametrical path of $G$. By Lemma 2.2, $H(G) \geq H(G-v)$. Therefore

$$
\begin{aligned}
H(G)-D(G) & \geq H(G-v)-D(G-v) \\
& \left.\geq \frac{5}{3}-\frac{n-1}{2} \quad \text { \{by induction }\right\} \\
& \geq \frac{5}{3}-\frac{n}{2} .
\end{aligned}
$$

Subcase 2.2: Let $D(G-v)=D(G)-1$. Let $u$ be the support vertex of $v$. If $N(u)$ has one vertex of degree at least two, we can prove by induction on $n$. We can easily check for unicyclic graph $G$ of order 7 such that $D(G-v)=D(G)-1$. Assume the result is true for all unicyclic graphs of order less than or equal to $n-1$. Let $G$ be a unicyclic graph of order $n$ and $v$ be the pendant vertex of the diametrical path of $G$. By Lemma 2.3,

$$
H(G) \geq H(G-v)+\frac{1}{2}
$$

Therefore

$$
\begin{aligned}
H(G)-D(G) & \geq H(G-v)+\frac{1}{2}-(D(G-v)+1) \\
& =H(G-v)-D(G-v)-\frac{1}{2} \\
& \left.\geq \frac{5}{3}-\frac{n-1}{2}-\frac{1}{2} \quad \text { \{by induction }\right\} \\
& =\frac{5}{3}-\frac{n}{2} .
\end{aligned}
$$

If $N(u)$ has two neighbours of degree at least two and $G$ has exactly one pendant vertex, then $G \cong U_{n}^{1}$. Now $H\left(U_{n}^{1}\right)=\frac{n}{2}-\frac{1}{5}$. If $n$ is odd, then $D\left(U_{n}^{1}\right)=\frac{n}{2}-\frac{1}{2}$. Hence $H\left(U_{n}^{1}\right)-D\left(U_{n}^{1}\right)=\frac{-7}{10} \geq \frac{5}{3}-\frac{n}{2}$. If $n$ is even, then $D\left(U_{n}^{1}\right)=\frac{n}{2}$. Hence $H\left(U_{n}^{1}\right)-D\left(U_{n}^{1}\right)=\frac{-1}{5} \geq \frac{5}{3}-\frac{n}{2}$.

If $N(u)$ has two neighbours of degree at least two and $G$ has more than one pendant vertex, then by Lemma 2.4 the result is true.

For proving the equality, assume that $H(G)-D(G)=\frac{5}{3}-\frac{n}{2}$. Since $D(G) \leq$ $n-2, \quad H(G)-(n-2) \leq H(G)-D(G)$, for all $G$. So our search is to find that $G$, 


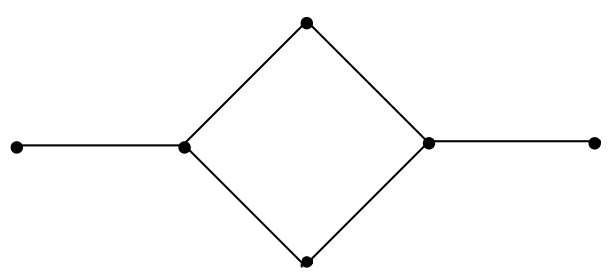

FiguRE $3 . G_{1}$

for which $D(G)=n-2$ and $H(G)-D(G)=\frac{5}{3}-\frac{n}{2}$. Among all unicyclic graphs with $D(G)=n-2$, the graph belongs to $\mathscr{C}$ is the only graph that satisfies the equality. Hence $G \in \mathscr{C}$. The converse is obviously true.

Remark 3.1. If $n \leq 6$ this lower bound is not true. One such graph is shown in Figure 3. For this graph $H\left(G_{1}\right)-D\left(G_{1}\right)=\frac{-7}{5}<\frac{-4}{3}=\frac{5}{3}-\frac{n}{2}$.

\section{REFERENCES}

[1] R. Balakrishnan and K. Ranganathan, A Textbook of Graph Theory, Springer-Verlog, New York, 2000.

[2] H. Deng, S. Balachandran, S. K. Ayyaswamy and Y. B. Venkatakrishnan, On the harmonic index and the chromatic number of a graph, Discrete Appl. Math. 161 (2013), 2740-2744.

[3] S. Fajtlowicz, On conjectures on graffiti-II, Congr. Numer. 60 (1987), 187-197.

[4] J. Liu, On harmonic index and diameter of graphs, J. Appl. Math. Phys. 1 (2013), 5-6.

[5] R. Wu, Z. Tang and H. Deng, A lower bound for the harmonic index of a graph with minimum degree at least two, Filomat $\mathbf{2 7}$ (2013), 51-55.

[6] L. Zhong, The harmonic index for graphs, Appl. Math. Lett. 25 (2012), 561-566.

[7] L. Zhong, The harmonic index on unicyclic graphs, Ars Combin. 104 (2012), 261-269.

${ }^{1}$ Department of Mathematics,

Holy Cross College,

TRICHY 620 002, INDIA

E-mail address: jermaths@gmail.com

${ }^{2}$ Department of Mathematics,

St. Joseph's College,

TRICHY 620 002, InDIA

E-mail address: benedict.mraj@gmail.com 